\title{
Collagen fiber arrangement in normal and diseased cartilage studied by polarization sensitive nonlinear microscopy
}

\author{
Jessica C. Mansfield \\ C. Peter Winlove \\ Julian Moger \\ University of Exeter \\ School of Physics \\ Stocker Road \\ Exeter, EX4 4QL \\ United Kingdom
}

\author{
Steve J. Matcher \\ University of Sheffield \\ The Kroto Institute \\ Broad Lane \\ Sheffield, S3 7HQ \\ United Kingdom
}

\begin{abstract}
Second harmonic generation (SHG) and two-photon fluorescence (TPF) microscopy is used to image the intercellular and pericellular matrix in normal and degenerate equine articular cartilage. The polarization sensitivity of SHG can be used directly to determine fiber orientation in the superficial 10 to $20 \mu \mathrm{m}$ of tissue, and images of the ratio of intensities taken with two orthogonal polarization states reveal small scale variations in the collagen fiber organization that have not previously been reported. The signal from greater depths is influenced by the birefringence and biattenuance of the overlying tissue. An assessment of these effects is developed, based on the analysis of changes in TPF polarization with depth, and the approach is validated in tendon where composition is independent of depth. The analysis places an upper bound on the biattenuance of tendon of $2.65 \times 10^{-4}$. Normal cartilage reveals a consistent pattern of variation in fibril orientation with depth. In lesions, the pattern is severely disrupted and there are changes in the pericellular matrix, even at the periphery where the tissue appears microscopically normal. Quantification of polarization sensitivity changes with depth in cartilage will require detailed numerical models, but in the meantime, multiphoton microscopy provides sensitive indications of matrix changes in cartilage degeneration. $\odot 2008$ Society of Photo-Optical Instrumentation Engineers. [DOl: 10.1117/1.2950318]
\end{abstract}

Keywords: cartilage; two-photon fluorescence microscopy; second-harmonic microscopy; intercellular; multiphoton microscopy.

Paper 07376R received Sep. 13, 2007; revised manuscript received Dec. 12, 2007; accepted for publication Feb. 2, 2008.

\section{Introduction}

2 Osteoarthritis is a widespread, painful, and debilitating joint 3 disease characterized by the degeneration and eventual loss of 4 the cartilage from the joint surface. Healthy cartilage has a 5 well-ordered structure, containing few cells, but an extensive 6 extracellular matrix comprising a network of collagen fibers 7 immersed in an amorphous, highly hydrated gel rich in pro8 teoglycans (see Fig. 1). This structure is lost as the disease 9 progresses, and many lines of research into its pathophysiol10 ogy and treatment are critically dependent on the development 11 of techniques for quantifying these changes. ${ }^{1,2}$ Particular in12 terest attaches to the organization of the network of collagen 13 fibers that are the principal determinant of the structure, and 14 associated mechanical properties that are central to the biome15 chanical function of the tissue and a number of physical meth16 ods have been applied to this problem. Polarized light micros17 copy, small and wide-angle x-ray scattering, and polarization 18 sensitive optical coherence tomography (PSOCT) have been 19 employed in a number of important and revealing studies. ${ }^{3-5}$ 20 The latter has the great attraction, because it can provide

Address all correspondence to: Jessica C. Mansfield, School of Physics, University of Exeter, Stocker Road, Exeter, EX4 4QL, United Kingdom. Tel: 441392 264142; Fax: 441392 264143; E-mail: j.c.mansfield@exeter.ac.uk depth imaging on tissue attached to subchondral bone, thus 21 opening the prospect of imaging in the intact joint. However, 22 the depth and lateral resolution of PSOCT are too low to 23 address many structural issues pertinent to cartilage patho- 24 physiology, and in the present work, we investigate the ability 25 of multiphoton microscopy to fulfill this need.

Multiphoton microscopy has been applied to biological 27 systems ranging from living cells ${ }^{6,7}$ to whole tissues. ${ }^{8,9}$ and 28 even living organisms, ${ }^{10}$ and it has a number of inherent ad- 29 vantages. Its nonlinearity allows the use of infrared excitation 30 wavelengths that maximize tissue penetration while maintain- 31 ing intrinsically high spatial resolution. ${ }^{11}$ It relies on two non- 32 linear processes that do not require the introduction of exog- 33 enous probe molecules or fixation and staining of the tissue. 34 The first is two-photon fluorescence (TPF). A number of en- 35 dogenous fluorophores have been identified in cells ${ }^{12}$ and, 36 particularly relevant to the present study, the extracellular 37 matrix, ${ }^{13}$ although their characterization is still incomplete. 38 The second mechanism, second harmonic generation (SHG), 39 occurs in molecules that do not possess inversion symmetry. It 40 is again fortunate for the study of the extracellular matrix that $\mathbf{4 1}$ collagen fibers are a strong source of SHG, although the 42

1083-3668/2008/13(4)/1/0/\$25.00 @ 2008 SPIE 


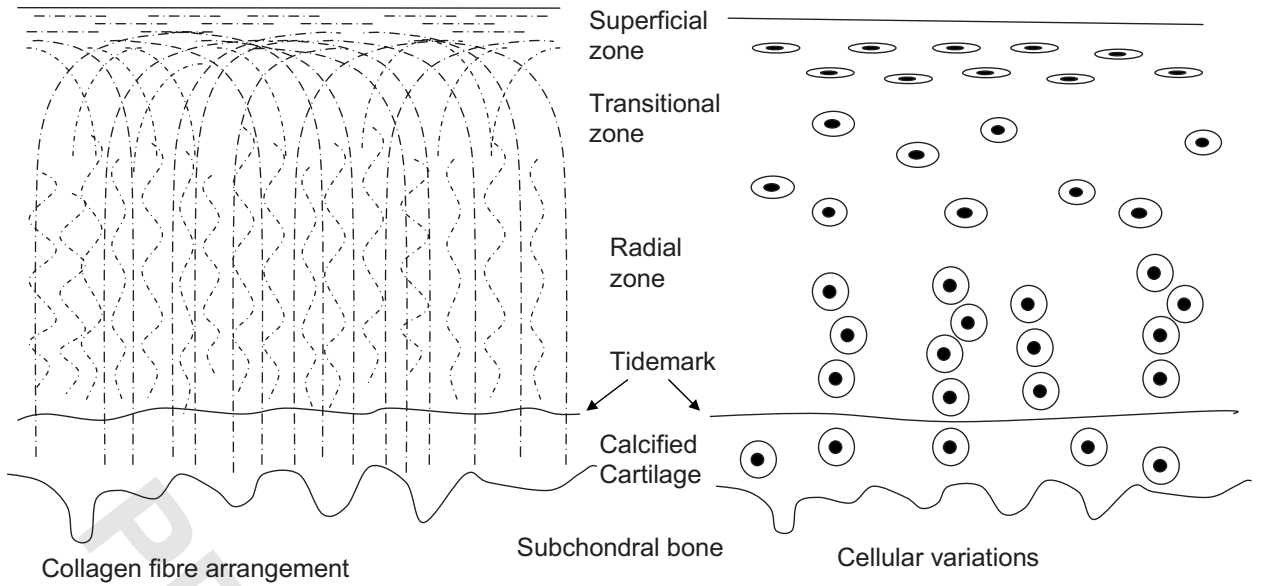

Fig. 1 A schematic diagram showing the different zones found in transverse sections of articular cartilage. The left-hand image represents the collagen fiber arrangements within the tissue, and the right-hand side shows changes in cell shape and arrangement that have been observed to occur with depth in the cartilage. Cartilage thickness is typically 1 to $4 \mathrm{~mm}$, depending on the species and joint examined.

45 mechanisms of its generation are still under debate. ${ }^{14,15}$ The 46 SHG signal is polarization sensitive and its intensity depends 47 on the angle between the fibers and the polarization of the 48 exciting radiation. This relationship has been explored in a 49 number of studies on rat tail tendon, ${ }^{15-19}$ a tissue that is com50 posed primarily of highly ordered fibrils of type-1 collagen. 51 To a first approximation, the maximum signal intensity is gen52 erated when the polarization is parallel with the fiber axis, and 53 the minimum intensity signal is generated when the polariza54 tion is perpendicular to the fiber axis. Therefore, polarization 55 sensitivity studies of SHG from tissues can provide informa56 tion on the fiber organization. ${ }^{20}$ Cartilage contains type- 2 col57 lagen, which has important but small biochemical differences 58 from type 1 and forms morphologically similar, but rather 59 finer, fibers with a complex organization that varies through 60 the depth of the cartilage, as shown in Fig. 1. This level of 61 organization would lead to the expectation that the SHG from 62 cartilage would be polarization sensitive, although a previous 63 study only detected in diseased tissue where the collagen fiber 64 organization had been disrupted. ${ }^{21}$

65 Further to explore this unexpected observation was the 66 starting point of the present investigation. Variations in SHG 67 polarization sensitivity with depth have previously been mea68 sured in tendons ${ }^{18,22}$ and other tissues such as dentine, dermis 69 and bone. ${ }^{22}$ The study investigates the polarization sensitivity 70 with depth in tendon and cartilage, and also considers the 71 effects of the biattenuance and birefringence of the overlying 72 tissue on the measure polarization sensitivity curve.

73 Our approach is based on comparisons of the variations in 74 the polarization sensitivity of TPF and SHG with depth. We 75 first study tendon as an example of a highly ordered collag76 enous structure whose birefringence and biattenuance have 77 been characterized in some detail. ${ }^{3,23}$ We then analyze normal 78 cartilage and lesions in the equine metacarpophalangeal joint. 79 This tissue was selected because its structure has been exten80 sively characterized by other methods, ${ }^{3,4}$ and the lesions that 81 develop spontaneously share many of the characteristics of 82 human disease. ${ }^{24}$

\section{Materials and Methods}

2.1 Methods

The experiments were carried using a home-built microscope, 85 whose essential features are illustrated in Fig. 2. A reflection 86 mode configuration was chosen, as this enabled the en-face 87 examination of cartilage samples still attached to the subchon- 88 dral bone, minimizing the disruption of the collagen architec- 89 ture.

90

Excitation was at $800 \mathrm{~nm}$ using a tuneable 100-fs pulsed 91 Ti:saphire laser with a repetition rate of $82 \mathrm{MHz}$ (Tsunami, 92 Spectra-Physics) The selection of the excitation wavelength 93 was based on a number of considerations. Previous studies 94 have produced conflicting results as to which wavelength 95 most efficiently excites SHG in collagen, ${ }^{7,25}$ but we found that 96 in our system, the signal of both SHG and TPF peaked be- 97 tween 780 and $820 \mathrm{~nm}$, and the use of longer wavelengths 98 increases tissue penetration and minimizes cellular damage. ${ }^{6} 99$ A 1.0-NA water dipping objective with a 2-mm working dis- 100 tance was employed (Fluor $60 \times$ ) and data acquisition was via 101 a photon-counting photomultiplier tube and a PC-based pho- 102 ton counting card (Hamamatsu H7360-02 and M8784). The 103 laser fundamental was removed by a short pass dichroic 104 beamsplitter (CVI Laser) and a bandpass filter (CVI Laser). 105 Blocking of the fundamental was greater than 9 OD and the 106 transmission of the TPF and SHG light about 30\%. To change 107 between SHG and TPF imaging, the filter directly below the 108 PMT was changed, with a 450 to $520-\mathrm{nm}$ bandpass filter be- 109 ing used for TPF detection and a 410 to 390-nm filter for 110 SHG detection (CVI Laser). For polarization measurements, 111 two 800-nm half wave plates were added to the system. The 112 first wave plate (CVI Laser) was placed before the dichroic 113 beamsplitter, orientated to ensure that completely p-polarized 114 entered the beamsplitter. The second (Meadowlark, Frederick, 115 Colorodo) was placed directly behind the back aperture of the 116 microscope objective to allow the polarization of the light 117 incident on the sample to be rotated. This arrangement was 118 necessary because our dichroic beamsplitter introduced differ- 119 


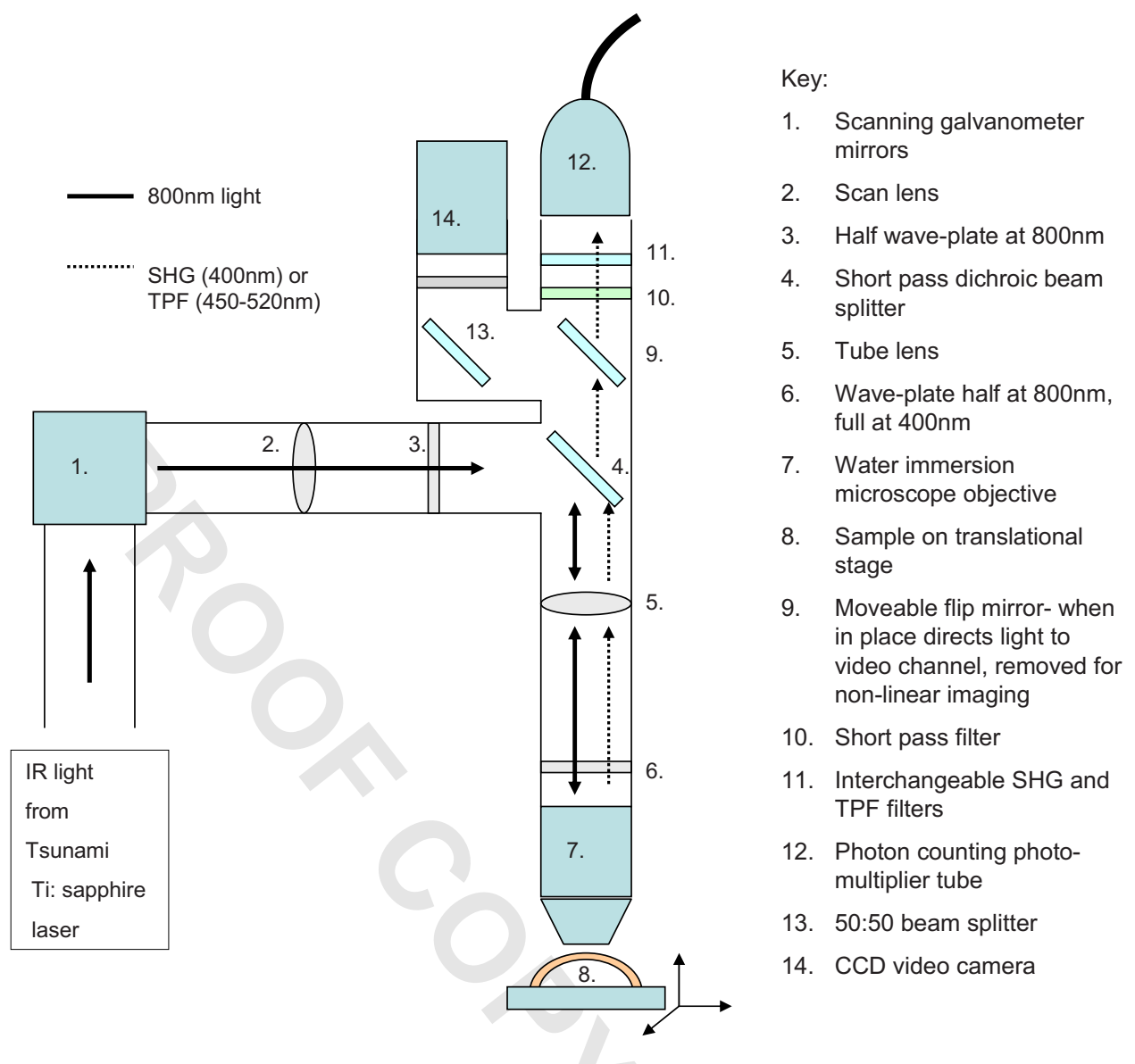

Fig. 2 The optical elements of the nonlinear microscope (not to scale).

120 ential phase-shifts between s and p-polarized states when used 121 in a reflection mode, which would convert the incident linear 122 polarization state to an elliptical state if the polarization was 123 rotated before the dichroic. The photon-counting PMT used 124 for the detection of the TPF and SHG signal was polarization 125 insensitive, and therefore the effect the wave-plate had on the 126 TPF light passing back through the objective would not affect 127 the experimental results.

128 Axial imaging was performed by raster scanning the beam 129 using two scanning galvanometer mirrors (Cambridge Tech130 nology Incorporated). An axial scan was generated by moving 131 the sample upward on a motor stage with an accuracy of 132 about $1 \mu \mathrm{m}$ (Physik Instrumente). The field of view of the 133 microscope objective allowed images up to $125 \times 125 \mu \mathrm{m}$ to 134 be acquired, the size being controlled by the voltage applied 135 to the galvanometer mirrors. The images reported in this study 136 were $100 \times 100 \mu \mathrm{m}$ and composed of $500 \times 500$ pixels. The 137 pixel dwell time was $10 \mu \mathrm{s}$.

138 The laser power used for imaging was approximately $13940 \mathrm{~mW}$ exiting the microscope objective. For the polarization 140 sensitivity measurements, a power of $30 \mathrm{~mW}$ was used for 141 acquisition of surface images, but power was increased with 142 scanning depth so that at $200-\mu \mathrm{m}$ depth, the power exiting 143 the objective was $140 \mathrm{~mW}$.

144 In polarization sensitivity experiments, the polarization of 145 the light incident on the sample was rotated in 10-deg steps 146 using the half wave plate behind the microscope objective.
The total intensity from the area scan was measured, and the 147 intensities for each angle were combined to create the polar- 148 ization sensitivity curve. This process was repeated at depth 149 steps into the tissue of $10 \mu \mathrm{m}$ in tendon and healthy cartilage 150 and $15 \mu \mathrm{m}$ in cartilage lesions. The depth steps were chosen 151 as a compromise between collecting a very detailed dataset 152 and minimizing the total scan time.

\subsection{Materials}

Tissue samples were obtained fresh from the abattoir, carti- 155 lage from the metacarpophalangeal joints and tendon from the 156 deep flexor tendon of horses. The age of the samples was 157 estimated by the abattoir staff and ranged from 5 to 20158 + years. Some samples were frozen prior to imaging, but this 159 had no discernable effect on the images. At the laboratory, the 160 joint was opened and a full depth plug of cartilage and several 161 millimeters of subchondral bone was excised from the apex of 162 the joint using a jeweller's saw. During measurement, samples 163 were maintained at room temperature and kept moist by im- 164 mersion in $0.15-\mathrm{M}$ saline.

\section{Results and Discussion}

The first stage of the investigation was to examine the TPF 167 and SHG signals generated from the surface layer of both 168 tendon and cartilage. In the case of cartilage, we explored the 169 variations in collagen fiber orientations within the imaging 170 


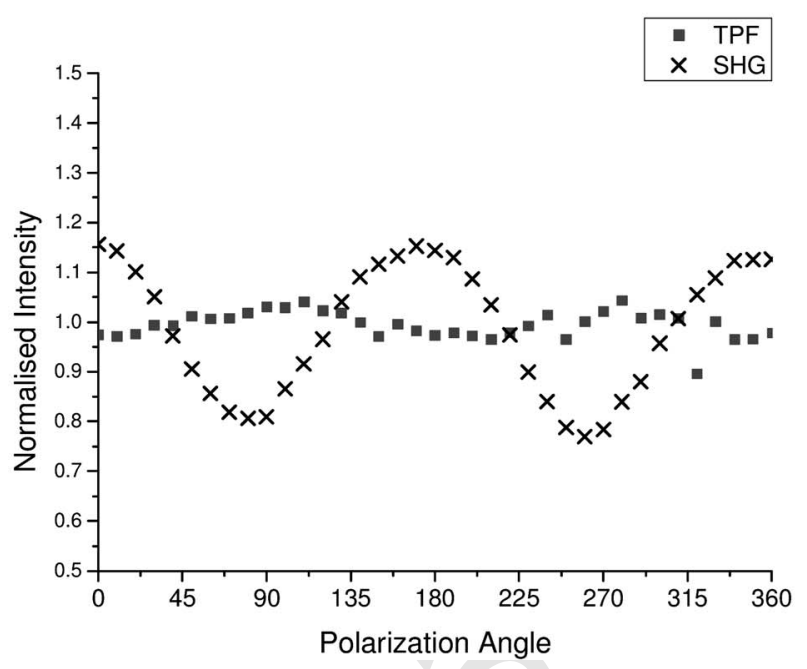

(a)

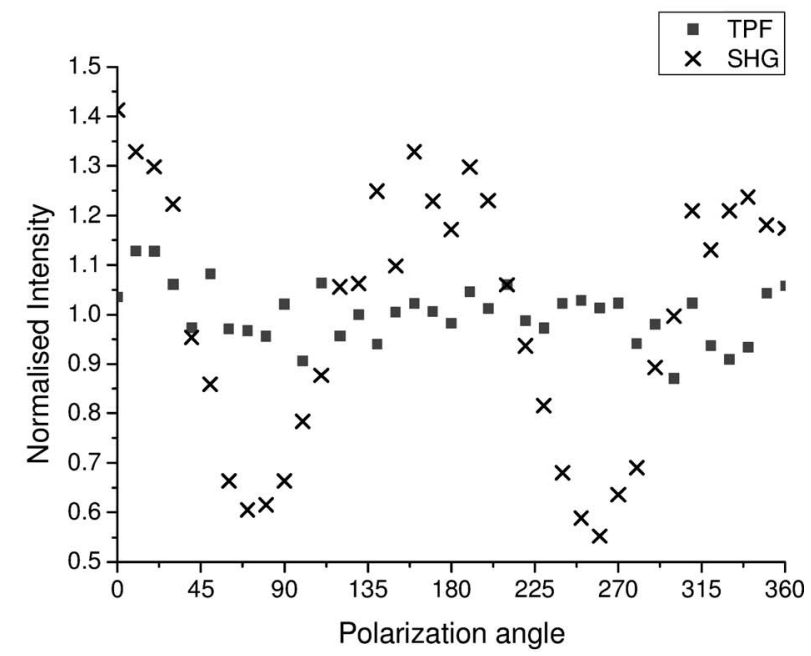

(b)

Fig. 3 Polarization sensitivity curves for both TPF and SHG taken at the surface in (a) cartilage and (b) tendon, with the $x$ axis showing the angle between the collagen fiber axis and the polarization of the laser fundamental. The intensities are normalized with respect to the mean intensity for each curve. Data were acquired at a depth of $10 \mu \mathrm{m}$ beneath the articular surface.

171 area by producing ratio images from the polarization sensitiv172 ity data. We then investigated signals emanating from deeper 173 in the tissue, first in tendon, which constitutes a simple model 174 system because its structure is uniform through the depth of 175 the tissue, and then in cartilage. The final section reports data 176 obtained from cartilage lesions.

\section{3.1 Surface Measurements on Cartilage and Tendon}

178 Figure 3 shows the angular variations in intensity of SHG and $179 \mathrm{TPF}$ at the surface of cartilage and tendon. The images were 180 acquired at a depth of $10 \mu \mathrm{m}$ from the surface zone of fibers 181 aligned parallel to the surface (Fig. 1). The polarization angle 182 is defined as the angle between the collagen fiber and the 183 polarization of the incident radiation, and in both tissues TPF 184 varied only weakly with angle. The visibilities $\left[\left(I_{\text {peak }}\right.\right.$ $\left.\left.185-I_{\text {trough }}\right) /\left(I_{\text {peak }}+I_{\text {trough }}\right)\right]$ for tendon and cartilage were $186(0.045 \pm 0.005)$ and $(0.04 \pm 0.02)$, respectively, (mean $\pm S D$, 187 measurements from five samples). Variations in the intensity 188 with polarization angle were comparable in magnitude with 189 the measurement noise. It therefore appears that TPF genera190 tion in both tissues does not have any significant intrinsic 191 polarization sensitivity, or the generation is polarization sen192 sitive but the sources are randomly orientated. As found by 193 previous researchers, ${ }^{15-19}$ the SHG signal from tendon showed 194 a strong polarization dependence, with the greatest intensity 195 when the laser excitation was parallel with the collagen fibrils. 196 The peak in the SHG signal corresponded with the long axis 197 of the collagen fibers visible in the SHG images to within $198 \pm 7 \mathrm{deg}$. The SHG signal from the cartilage surface also de199 pended on polarization and had a period of 180 deg, suggest200 ing a preferred orientation of the collagen fibers in the super201 ficial zone of the cartilage. Though this was not observed in 202 previous studies on SHG from cartilage, ${ }^{21}$ it is consistent with 203 well-established histological evidence. ${ }^{1,26}$

204 The visibility of the intensity-polarization angle plots was 205 calculated as a measure of the degree of collagen fiber orga206 nization in the tissue. For tendon, the visibility was
$0.29 \pm 0.04$, and for cartilage the visibility was $0.11 \pm 0.05^{207}$ (mean $\pm \mathrm{SD}$, measurements from six samples). These differ- 208 ences are consistent with the perceived differences in the level 209 of collagen fiber organization in the two tissues, but different 210 levels of intrafibrillar organization or optical properties be- 211 tween type- 1 and -2 collagen may also contribute.

To investigate whether there were differences in collagen 213 fiber organization within the $100-\mu \mathrm{m}^{2}$ scanning area, we 214 compared SHG images with the polarizer set to provide maxi- 215 mum signal with those with the polarizer rotated through 216 $90 \mathrm{deg}$. Representative images and the resulting ratio image 217 are shown in Fig. 4. Histological studies have described the 218 pericellular matrix as basket-like and containing finer col- 219 lagen fibrils. ${ }^{1,26,27}$ Therefore, we were expecting less polariza- 220 tion sensitivity and consequently a ratio close to 1 in these 221 regions. However, these differences were not apparent in the 222 ratio image, and although the ratio varied between 0.5 and 2223 on a length scale of a few microns, the variations showed no 224 obvious correlation with position. On the basis of these obser- 225 vations, it was felt that averaging over a $100 \times 100-\mu \mathrm{m}$ im- 226 age area was suitable for subsequent polarization sensitivity 227 investigations. A smaller area will provide information on the 228 collagen fiber arrangement on lower hierarchical levels in- 229 stead of the zonal architecture described in Fig 1. Averaging 230 over longer length scales is not required, as the polarization 231 sensitivity results averaged over the $100 \times 100-\mu \mathrm{m}$ image 232 area are repeatable, and therefore a larger imaging area will 233 produce the same results.

\subsection{Depth Variations in Tendon}

To determine how the effects of biattenuance and birefrin- 236 gence in the overlying tissues affected measurements of 237 changes in polarization sensitivity with depth, we undertook 238 an initial study on tendon, whose structure and fiber orienta- 239 tions are constant with depth and can be observed directly in 240 the multiphoton images. The angular dependence of SHG and 241 TPF was measured at depths up to $200 \mu \mathrm{m}$ and values aver- 242 

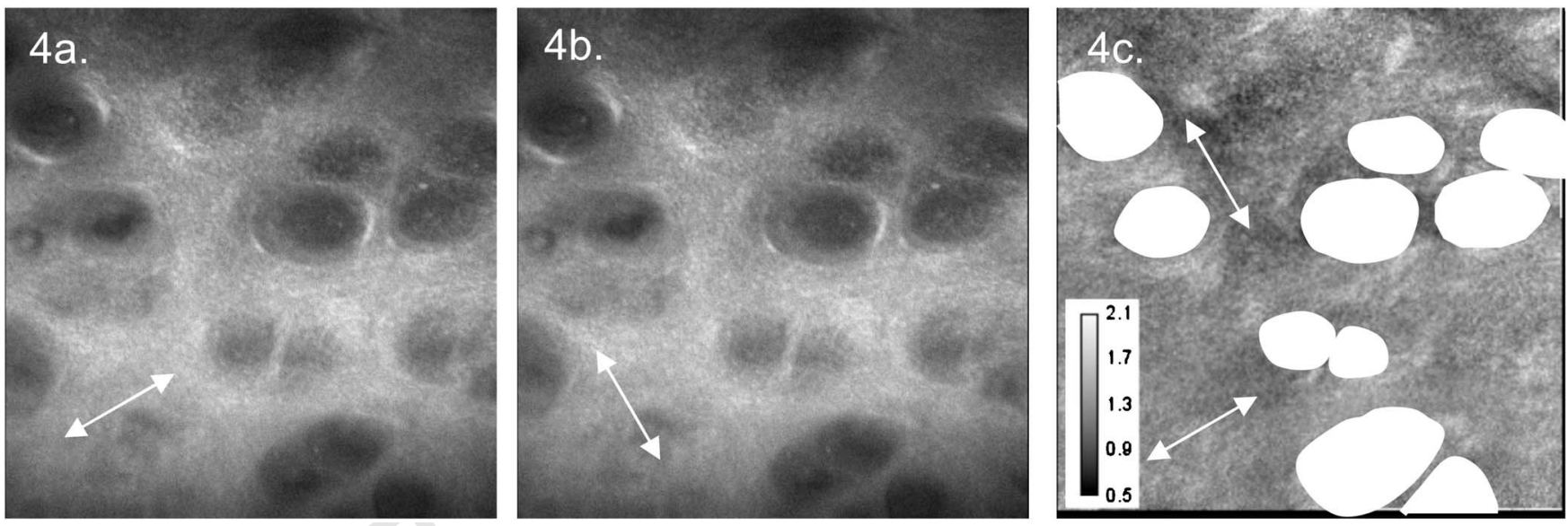

Fig. 4 SHG images acquired with the polarizer at the position of (a) maximum intensity and (b) rotated through 90 deg, with the arrows indicating the polarization angle. (c) shows the ratio of the two images. The dark regions in (a) and (b) represent the locations of the chondrocytes. In the ratio image, these areas are circled. (Field $100 \times 100 \mu \mathrm{m}$, arrows indicate the polarization of the laser light.) The dark region in the top right quarter of the image represents an area where the polarization sensitivity varies from that found in the rest of the scan area.

243 aged over a $100-\mu \mathrm{m}^{2}$ field in three samples are summarized 244 in Fig. 5. Although the orientation of the collagen fibers re245 mained constant with depth, the SHG polarization sensitivity 246 pattern changed dramatically with depth, as is shown in Fig. 247 5(a). The TPF polarization sensitivity in Fig. 5(b) shows an 248 increase with depth, with strong peaks in intensity appearing 249 perpendicular to the collagen fiber orientations. This indicates 250 that the optical properties of the overlying tissue are contrib251 uting to the measured patterns. Figures 5(c) and 5(d) show the 252 natural $\log$ of the unnormalized data for the SHG and TPF 253 polarization, respectively. These plots have been included be254 cause they show the attenuation of the light through the tissue. 255 From the TPF data, it was possible to estimate an upper 256 bound for the biattenuance of the tissue at the excitation 257 wavelength. Surface imaging demonstrated that the TPF exci258 tation efficiency has no intrinsic polarization sensitivity, 259 which in turn suggests an isotropic orientation of fluorescence 260 excitation dipoles within the focal volume. The variation in $261 \mathrm{TPF}$ emission at depth can thus be presumed to arise from 262 "biattenuance" (a term introduced by Kemp et al. ${ }^{23}$ to describe 263 differential absorption or scattering of the excitation and/or 264 emitted light for different incident polarization states). Let us 265 assume that the superficial layer contains a well-defined trans266 mission axis such that field strengths of light linearly polar267 ized parallel and perpendicular to this axis experience a dif268 ferential attenuation coefficient given by $2 \pi \Delta \chi / \lambda_{0}$, where $269 \Delta \chi$ is the biattenuance of the medium. Further, let us assume 270 that these axes are coincident with the birefringence fast and 271 slow axes of the superficial layer, i.e., we effectively assume 272 that the superficial tissue can be described by a complex bi273 refringence $\Delta n+i \Delta \chi$. Incident linearly polarized light aligned 274 with either of these directions will thus remain polarized 275 along these directions with increasing depth, and the differen276 tial attenuation of the excitation light between these directions 277 will attain the maximum value. Linearly polarized light inci278 dent at other angles will undergo conversion to elliptical po279 larization states with increasing depth, and will thus experi280 ence an intermediate degree of attenuation. We thus identify 281 the angles at which maximum and minimum TPF intensities occur as defining the directions of the fast and slow axes. ${ }^{282}$ Recalling that our detection system is polarization insensitive, 283 if the emitted TPF were itself unpolarized, then the emitted 284 light would propagate to the detector with equal efficiency, 285 regardless of the polarization direction of the excitation light. 286 We could then use the difference in the maximum and mini- 287 mum TPF intensities to infer $\Delta \chi$ at the excitation wavelength 288 a follows.

Following Kemp et al., ${ }^{23}$ we note that $\Delta \chi$ defines the ratio 290 of the field strengths polarized along the two axes at a depth 291 $\Delta z$, such that

$$
\frac{E_{\max }}{E_{\min }}=\exp \left(\frac{2 \pi \Delta \chi}{\lambda_{0}} \Delta z\right)
$$

Noting the TPF intensity scales as the fourth power of the 294 field, the ratio of the emitted TPF fluxes is then described by 295

$$
\frac{I_{\max }}{I_{\min }}=\exp \left(\frac{8 \pi \Delta \chi}{\lambda_{0}} \Delta z\right)
$$

Thus, if the tissue has biattenuance, the ratio of the maximum 297 to minimum intensity should increase exponentially with 298 depth and a plot of $\ln \left(I_{\max } / I_{\min }\right)$ versus $\Delta z$ would have a 299 slope of $8 \pi \Delta \chi / \lambda_{0}$, where $\Delta \chi$ unambiguously represents the 300 biattenuance at the excitation wavelength, i.e., $800 \mathrm{~nm}$. We 301 see from the data [Fig. 5(e)] that an exponential increase can 302 be fitted to the first $100 \mu \mathrm{m}$ of the curves, but beyond this, 303 the ratio remains constant or falls. The exponential increase 304 appears quasi linear over this range due to the low value for 305 the biattenuance of collagen. This may be because at these 306 depths, the excitation light becomes depolarized due to scat- $\mathbf{3 0 7}$ tering in the tissue, ${ }^{28}$ or the collagen fibers are no longer per- 308 fectly aligned. However, restricting the analysis to the most 309 superficial $100 \mu \mathrm{m}$, we fitted the data to the equation 
Mansfield et al.: Collagen fiber arrangement in normal and diseased cartilage...

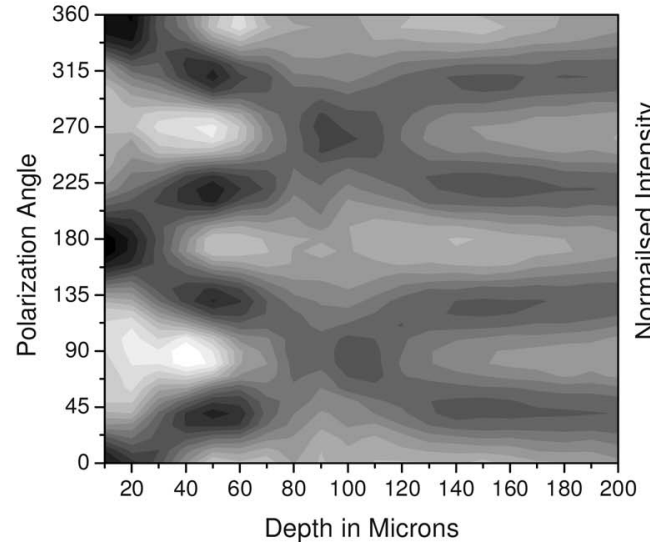

(a)

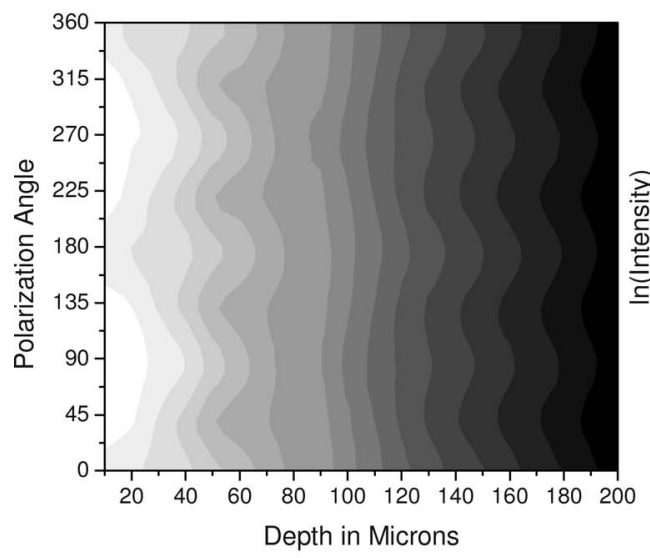

(c)
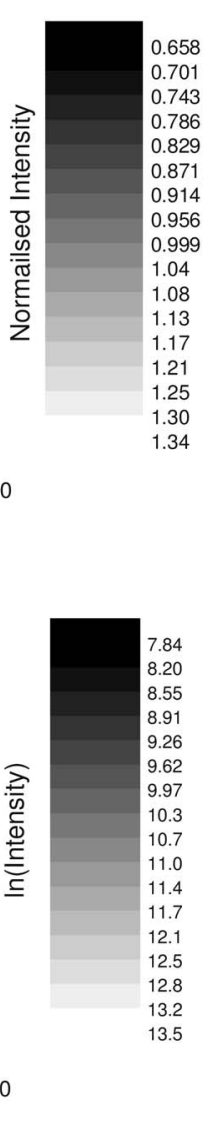
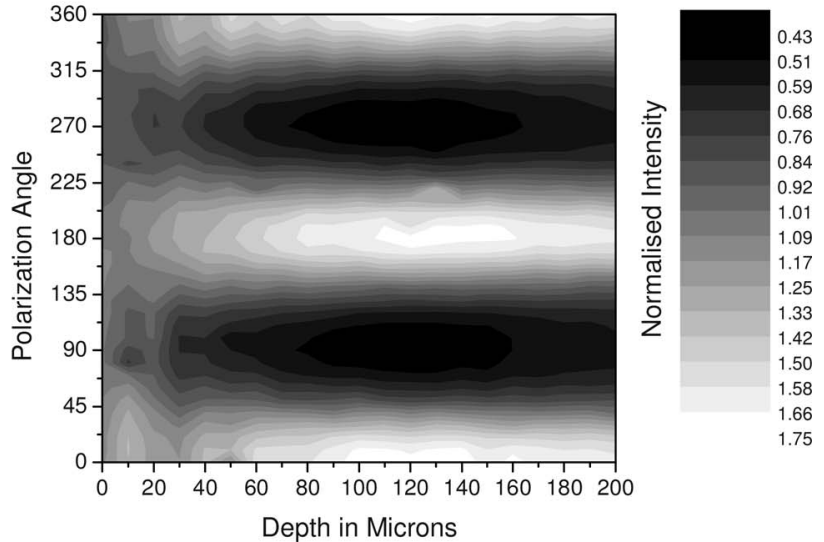

(b)
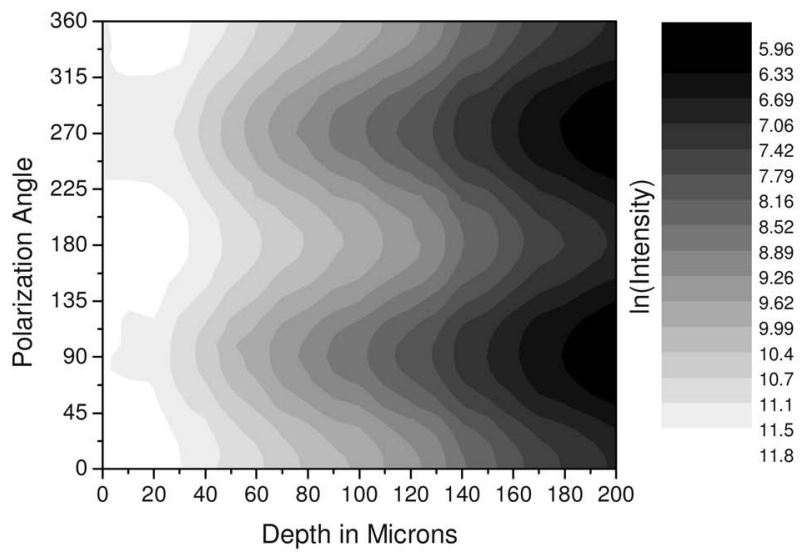

(d)

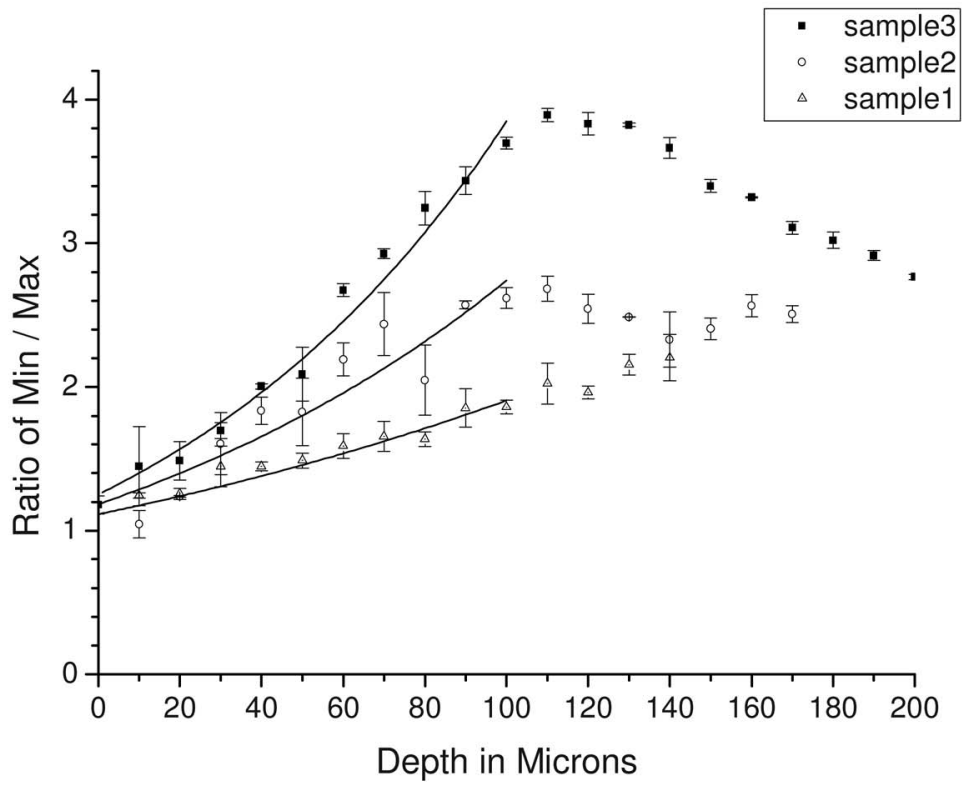

(e)

Fig. 5 Polarization dependence of (a) SHG and (b)TPF in tendon at different depths [collagen fibers orientated at 90 deg to the original incident beam polarization $(0 \mathrm{deg})$, intensities normalized by the mean intensity of the image field at each depth]. (c) and (d) show the natural log of the unnormalized polarization sensitivity. (e) shows the ratio of maximum intensity of the TPF/ the minimum intensity of the TPF as a function of depth in the tissue. 


\section{PROOF COPY 007804JBO}

Mansfield et al.: Collagen fiber arrangement in normal and diseased cartilage...

311

$$
\frac{I_{\max }}{I_{\min }}=\exp \left[\frac{8 \pi \Delta \chi}{\lambda_{0}}\left(z-z_{0}\right)\right]
$$

312 where $z$ is the depth into the tissue, $z_{0}$ is a factor to allow for 313 any uncertainty in identifying the surface of the tendon, $\lambda_{0}$ is 314 the wavelength of the incident light, and $\Delta \chi$ is the biattenu315 ance using Microcal Origin. This gave a biattenuance of $3162.65 \times 10^{-4}$ (range $1.7-3.6 \times 10^{-4}, \mathrm{R}^{2}>0.85$ for all fits), 317 which is comparable with values measured for different types 318 of tendon using polarization sensitive OCT (rat tail tendon $3195.3 \times 10^{-4}$, rat Achilles tendon $1.3 \times 10^{-4}$, chicken patel320 lofemoral tendon $\left.2.1 \times 10^{-4}\right){ }^{23}$

321 These estimates represent only an upper bound for the bi322 attenuance, however, because even with an isotropic distribu323 tion of fluorophores, the TPF may still be partially polarized. 324 This is because the incident polarization vector will most ef325 ficiently excite parallel-aligned dipoles, resulting in the TPF 326 being partially polarized in the direction of the incident laser 327 polarization (unless the fluorophores rotate on a time scale 328 fast compared with the fluorescence lifetime, which is un329 likely in collagen). ${ }^{29}$ This means that the emitted light will be 330 transmitted to the detector with different efficiencies for the 331 two orthogonal polarization states, so that the intensity ratio 332 will then depend on the value of $\Delta \chi$ at both the excitation and 333 emission wavelengths. The precise relationship between our 334 value and the true values at 400 and $800 \mathrm{~nm}$ will depend on 335 the degree of fluorescence anisotropy. This highlights the need 336 for further research into not only identifying but also charac337 terizing the properties of endogenous two-photon fluoro338 phores.

339 For the SHG, the pattern of polarization with depth in ten340 don represents the combined effects of the biattenuance, bire341 fringence, and the intrinsic polarization sensitivity of the col342 lagen SHG. Tendon is birefringent, with a higher refractive 343 index for light polarized parallel to the collagen fibers. ${ }^{19}$ The 344 birefringence $(\Delta n)$ is approximately 0.0045 for equine flexor 345 tendon, ${ }^{3}$ indicating that a $180-\mu \mathrm{m}$ thickness of tendon would 346 act as a whole wave plate at $800 \mathrm{~nm}$. It should be possible to 347 use this information to recreate our polarization sensitivity 348 patterns, using either a Jones or a Muller matrix (if scattering 349 is found to be a significant effect) to describe the effects of the 350 overlying tissue, and the Freund mode ${ }^{16}$ to describe the po351 larization sensitivity of collagen fibers. However, the effects 352 of beam geometry, such as varying interaction lengths and 353 ray-to-fiber inclination angles across the beam profile, are 354 likely to be significant for the large numerical aperture objec355 tive we employed, and it would be a nontrivial task to include 356 these in the calculations. Such studies will form the basis of 357 further work to be reported in the future.

\section{3.3 Depth Variations in Cartilage}

359 TPF and SHG polarization sensitivity data were taken on the 360 same field in samples of healthy cartilage, and representative 361 plots are shown in Fig. 6. It proved possible to acquire data at 362 depths up to $200 \mu \mathrm{m}$. This region is marked on the histologi$363 \mathrm{cal}$ section taken of the sample after imaging [Fig. 6(e)]. From 364 this we can see it corresponds to the superficial and transi365 tional zones shown in Fig. 1. The TPF polarization measure366 ments were affected by photobleaching, and although expo367 sure time was kept to a practical minimum, it was impossible entirely to eliminate the effect. The photobleaching in carti- ${ }^{368}$ lage was a more significant effect than in tendon. This may 369 indicate that a different fluorophore is responsible for the fluo- $\mathbf{3 7 0}$ rescence in the two tissues, or that the differences in the ex- 371 tracellular matrix environment effect the photobleaching rates. 372 In contrast to tendon, the TPF images did not show strong 373 polarization sensitivity. At 150 to $200 \mu \mathrm{m}$, four peaks were 374 evident but their visibility was very small $(<0.05)$ compared 375 to that in tendon $(\sim 0.6)$ at a similar depth. It is therefore 376 apparent that biattenuance is not as significant in cartilage as $\mathbf{3 7 7}$ it is in tendon, and the structural bases of this difference re- 378 quire further investigation. If this is interpreted as a null result $\mathbf{3 7 9}$ for detecting biattenuance in cartilage, we can place an upper 380 bound for the possible biatteunance of $6 \times 10^{-5}$ by assuming 381 that periodic variations should be at least two times greater 382 than the variations due to noise or photobleaching. 383

The variation in SHG polarization with depth showed a 384 clear repeatable pattern in all the normal samples of cartilage 385 investigated. At the surface of the tissue, there were two in- 386 tensity maxima, but at greater depths two additional peaks 387 occurred between the original peaks. There was also an in- 388 crease in visibility of the peaks with depth into the tissue. The 389 depth at which the additional peaks appeared varied between 390 60 and $90 \mu \mathrm{m}$ in different samples.

391

Relating the changes in polarization sensitivity with depth 392 to fiber orientation is a more complex problem in cartilage 393 than in tendon. In contrast to tendon, the birefringence and 394 biattenuance properties of the tissue are likely to change with 395 depth, and this level of complexity cannot simply be de- 396 scribed by a single Jones or Muller matrix. In addition, the 397 collagen fibers form a 3-D network, so the fibers in the deeper 398 zones no longer lie in the imaging plane, and the larger the 399 angle between the collagen fiber and the imaging plane, the 400 weaker the polarization dependence and the weaker the SHG 401 signal. ${ }^{19}$ It seems therefore that it will be necessary to develop 402 a numerical model, perhaps based on measurements of biat- 403 tenuance and birefringence, in a series of thin slices of carti- 404 lage at increasing depth into the tissue.

\subsection{Cartilage Lesions}

Spontaneous lesions were found on the apex of a large num- 407 ber of the metacarpophalangeal joints examined. The histol- 408 ogy of these lesions has been discussed in detail elsewhere. ${ }^{30} 409$ We report here on the detailed examination of three such le- 410 sions.

Figure 7 shows paired TPF and SHG images taken from 412 the surface of the cartilage in the histologically normal tissue 413 close to the lesion, at the periphery of the lesion, and in the $\mathbf{4 1 4}$ center of the lesion. The former images are very similar to 415 those described earlier and reported by previous authors. ${ }^{21,31} 416$ At the periphery of the lesion, where the histology showed an $\mathbf{4 1 7}$ intact surface but a loss of zonal structure, the TPF images 418 appeared normal, but the SHG images showed abnormally 419 high signal intensities from the pericellular matrix. In the core 420 of the lesion, there were far fewer cells and the matrix also 421 appeared abnormal with a rippling effect clearly visible. The $\mathbf{4 2 2}$ rippling appears more strongly in the SHG than the TPF and 423 so can be confidently ascribed to a collagen abnormality. Ra- 424 tio images emphasized the difference in the pericellular ma- 425 trix between the normal tissue and the tissue peripheral to the 426 


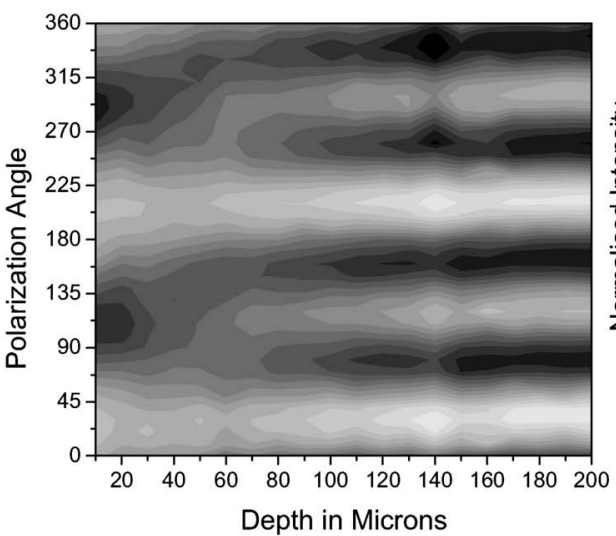

(a)

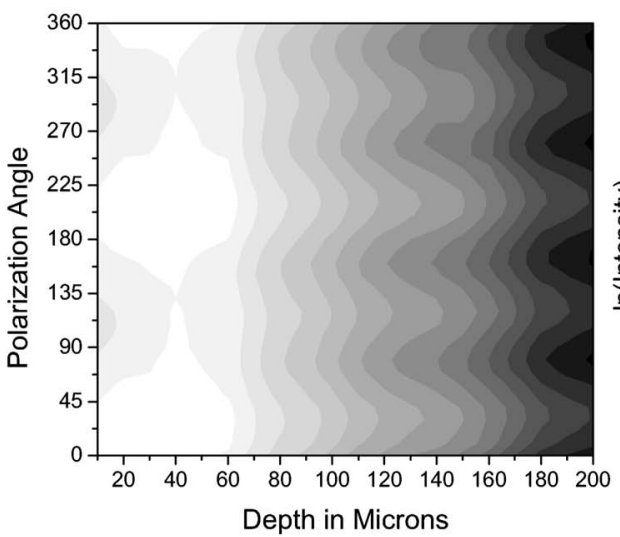

(c)
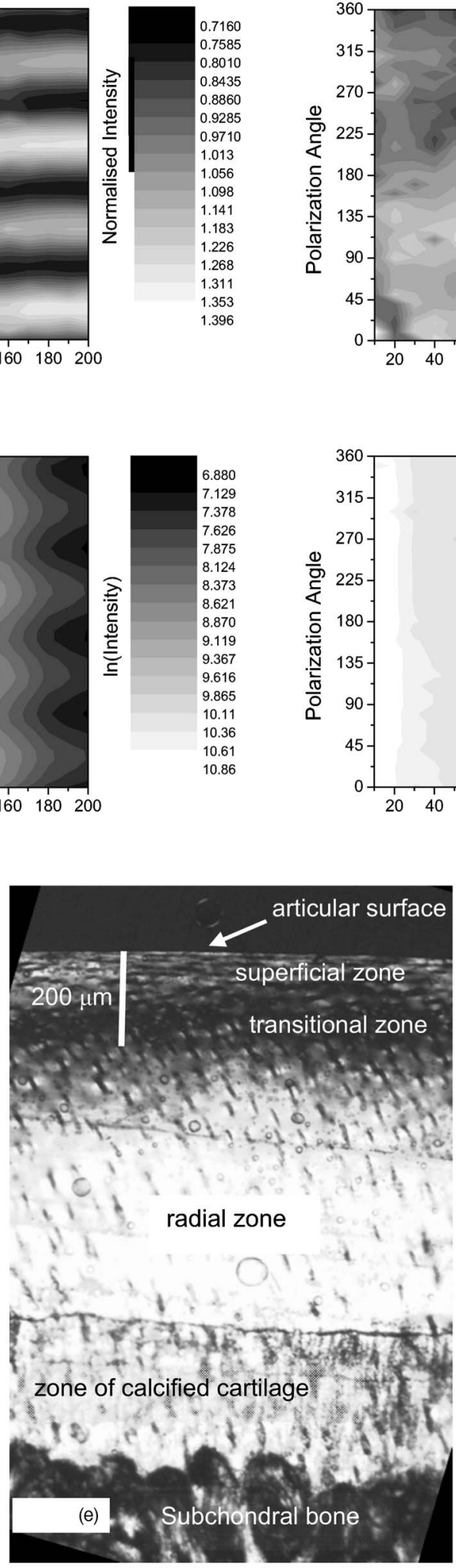

(e)

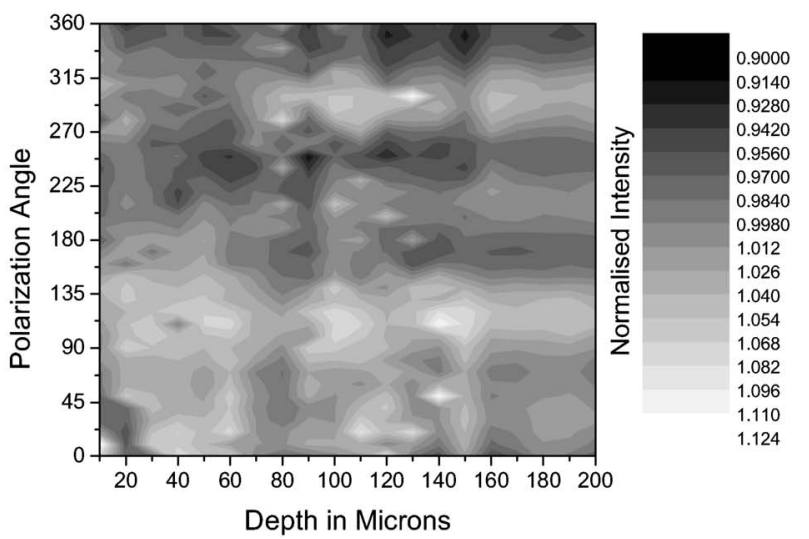

(b)

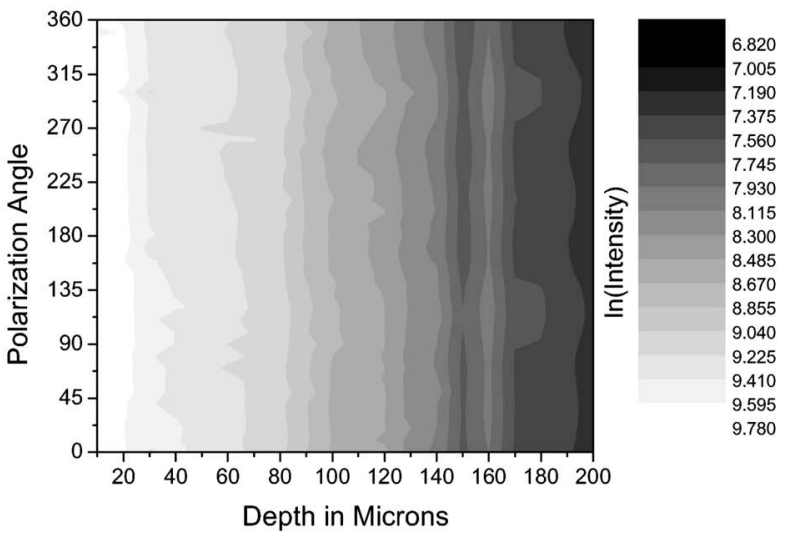

(d)

Fig. 6 Polarization sensitivity with depth in cartilage, (a) SHG and (b) TPF (intensities normalized by the mean field intensity at each depth). (c) and (d) show the natural log of the unnormalized polarization sensitivity for SHG and TPF. (e) shows a histological section taken from the sample after the polarization sensitive measurements, viewed through crossed polarizers to reveal collagen organization. 


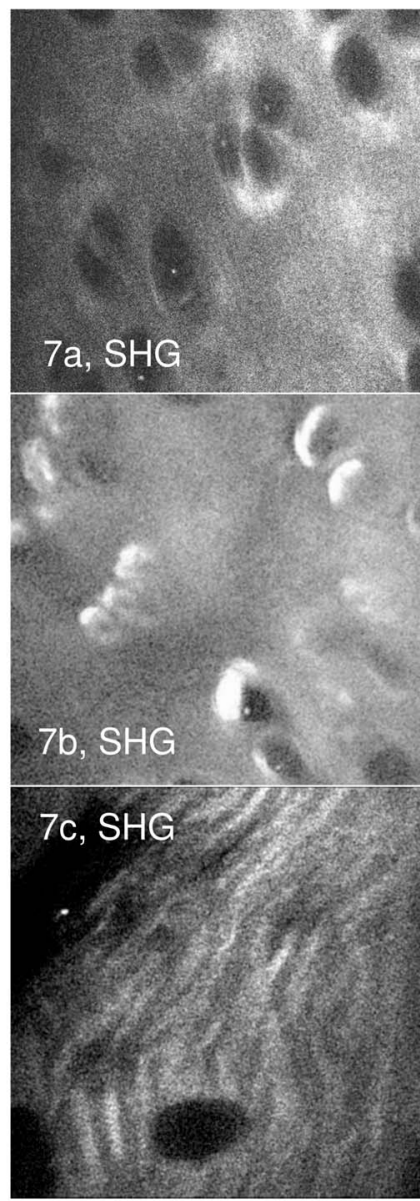

Region with healthy looking matrix and cells in the TPF and SHG images similar to those shown in shown in $7 a$

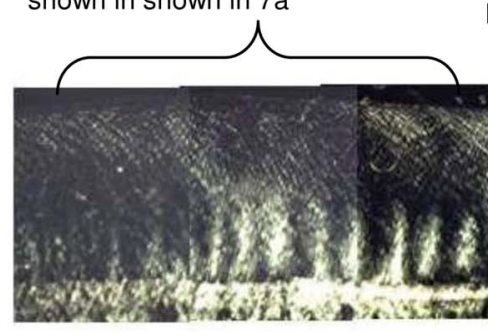

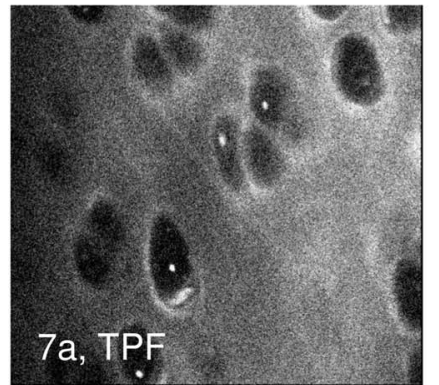
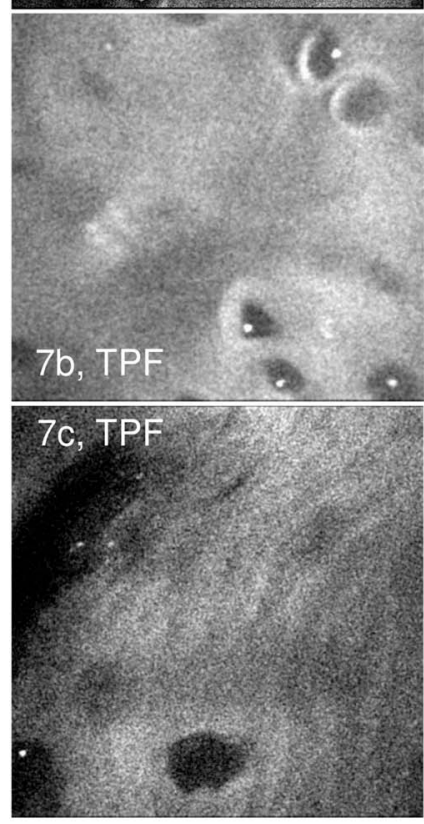
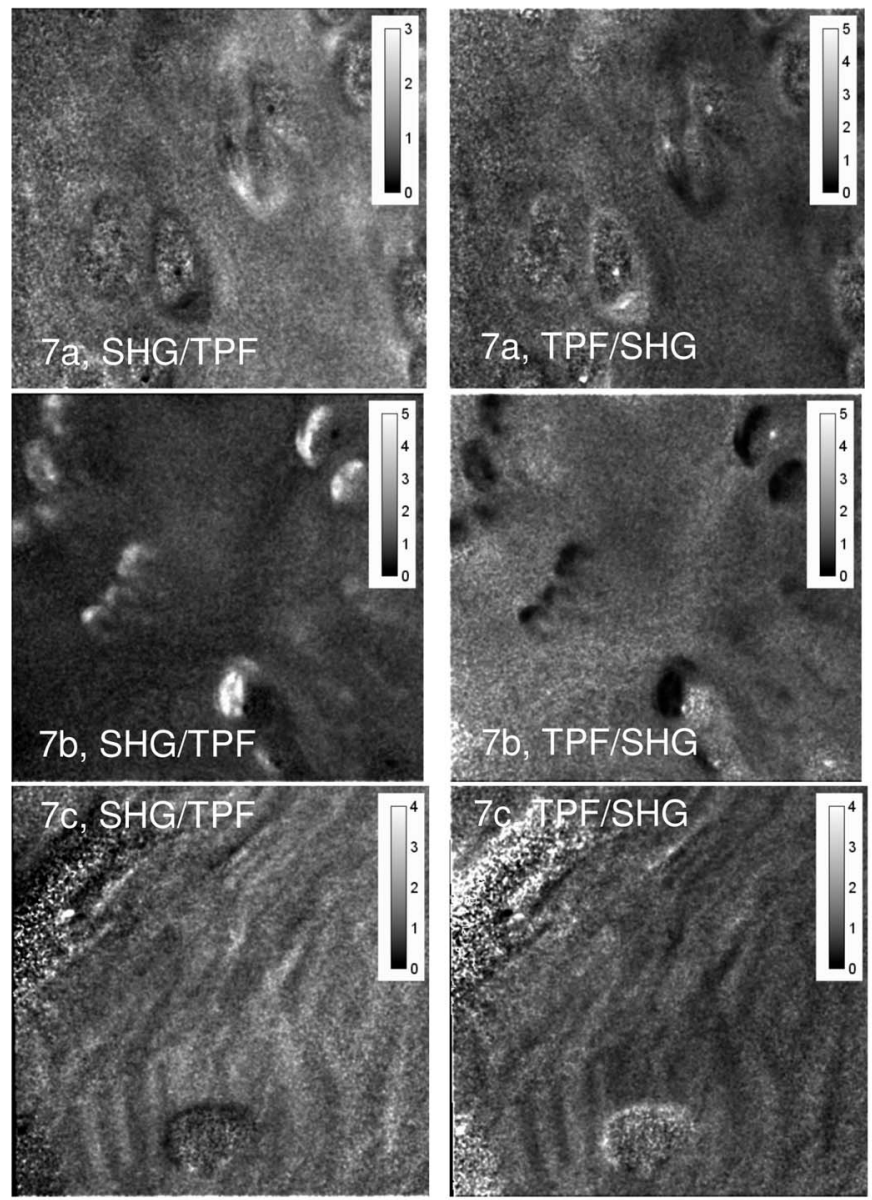

Lesion area: very strong birefringence due to high collagen content: images in the SHG and TPF show very few cells rippled lookina matrix in the SHG as see 7c.

Region nearing the lesion histology shows normal arcade structure of matrix is being lost: SHG and TPF
images show unusual looking cells see $7 \mathrm{~b}$.

7d. Histological slice of the lesion viewed between crossed polarizers

Healthy region of

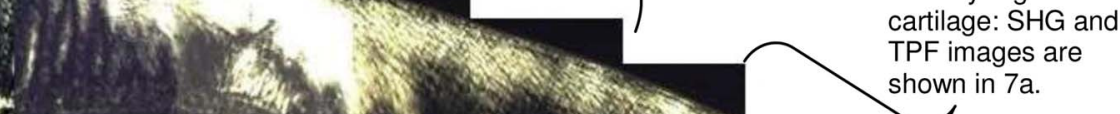
9. 1 (1) 1

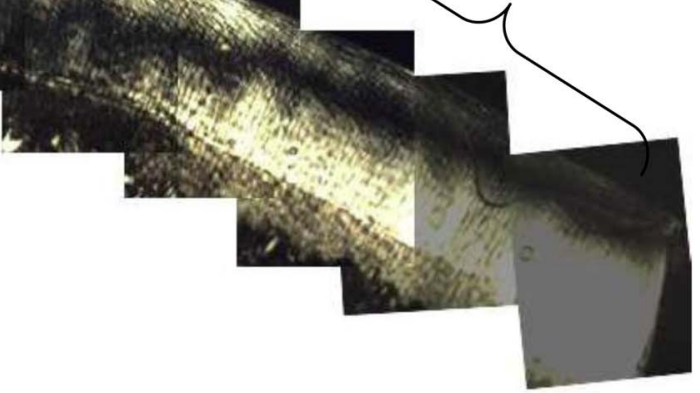

Fig. 7 Cartilage lesion. (a) SHG and TPF images taken from a region $3 \mathrm{~mm}$ away from the center of the lesion, which appeared normal in histological sections. (b) Images from the periphery of the lesion, showing abnormalities in the SHG from the pericellular matrix, while the TPF image remains normal. (c) Images from the center of the lesion, showing loss of cells and rippling effect in the SHG image. (Field 100 $\times 100 \mu \mathrm{m}$ ). The ratio images shown in the right-hand column highlight the changes in (b) the pericellular matrix and that (c) the rippling effect is stronger in the SHG image. 


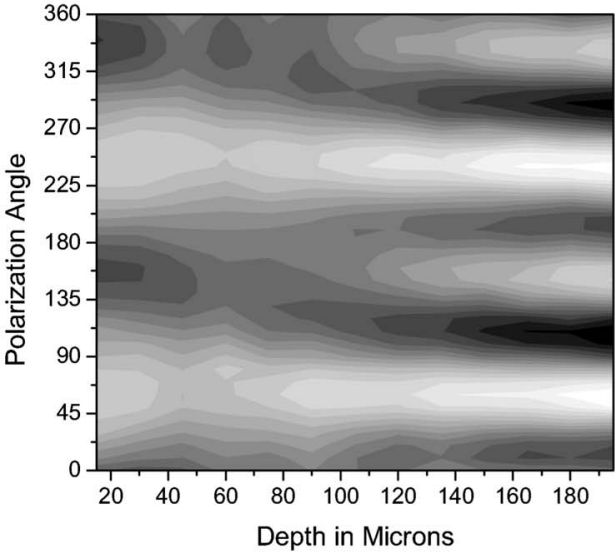

(a)

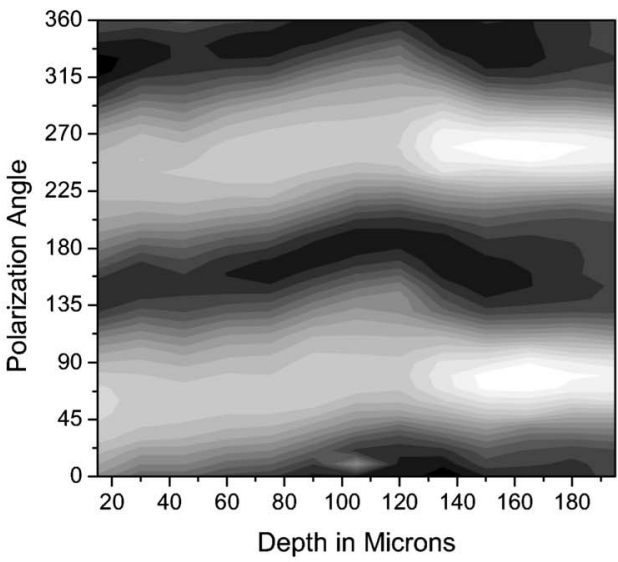

(c)

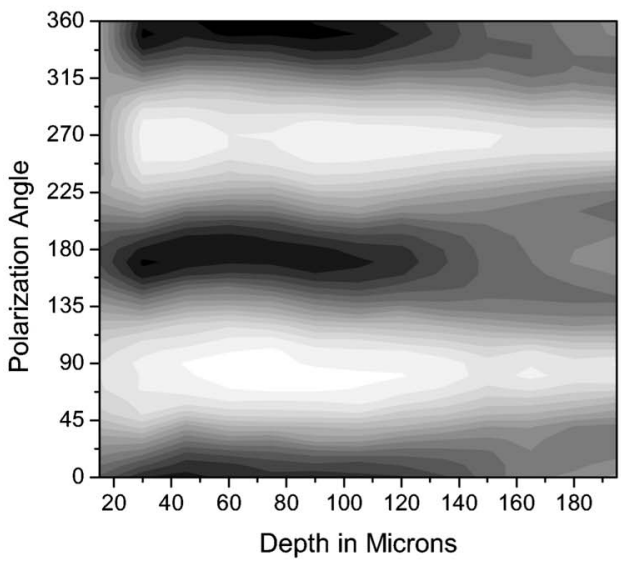

(e)
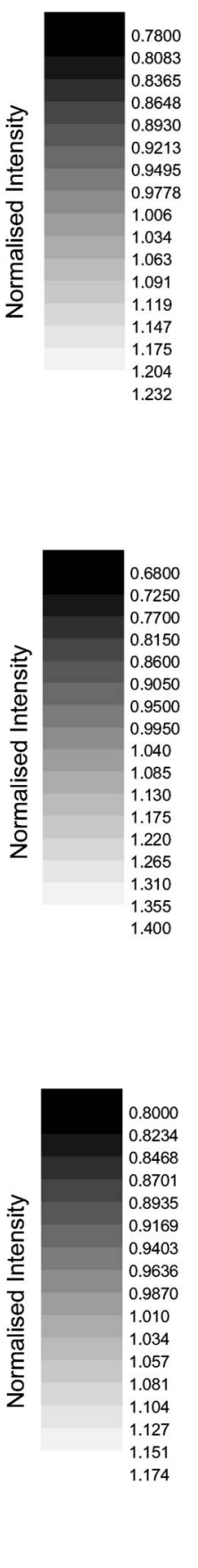
0.8000
0.8234
0.8468
0.8701
0.8935
0.9169
0.9403
0.9636
0.9870
1.010
1.034
1.057
1.081
1.104
1.127
1.151
1.174
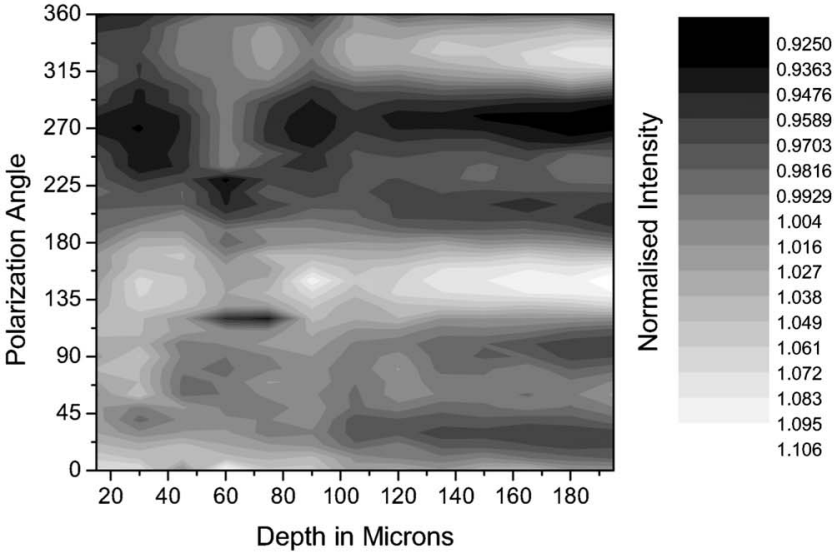

(b)
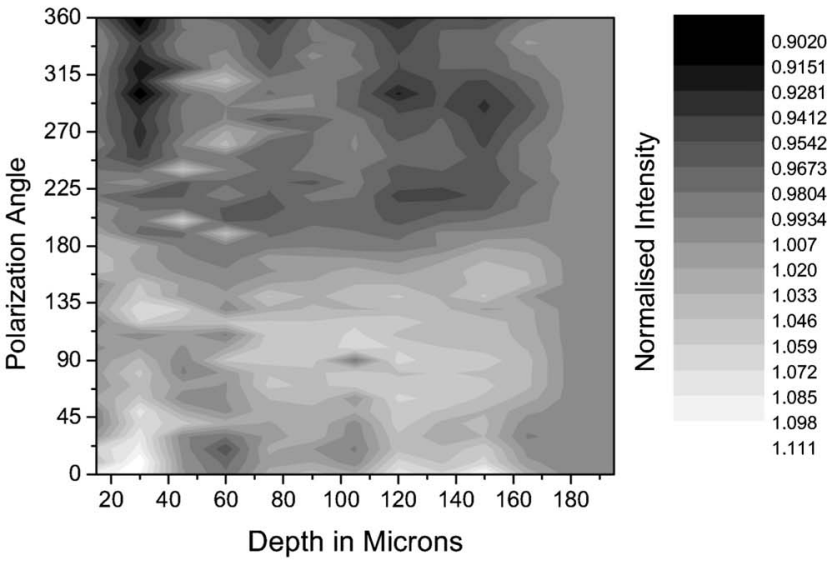

(d)
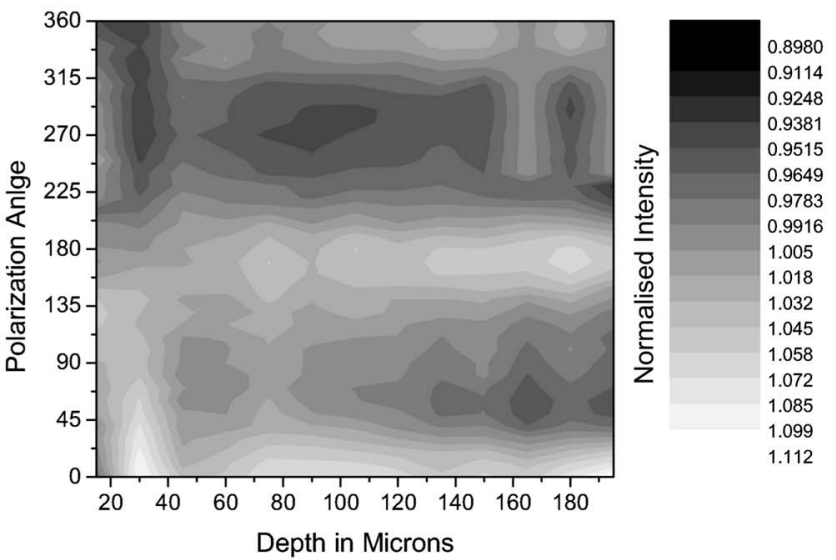

(f)

Fig. 8 The polarization dependence of TPF and SHG in the vicinity of a lesion, with site 1 being macroscopically normal-looking tissue close to the lesion, site 2 being approximately central to the lesion and site 3 being tissue in the periphery of the lesion.

427 lesion, with the ratio of SHG/TPF much greater in the peri428 cellular matrix at the periphery of the lesion.

429 Figure 8 shows the measurements of both SHG and TPF 430 polarization as a function of depth, in the core of the lesion 431 and at the periphery, in macroscopically normal tissue. At the 432 latter sites, the tissue showed the weak biattenuance in the 433 TPF signal reported earlier, but at the core of the lesion, no 434 polarization sensitivity was observed. Again we interpret this 435 as a null result and place an upper bound of $6 \times 10^{-5}$ on the biattenuance, as for the TPF from healthy cartilage.

The SHG depth profiles showed larger changes than the 437 TPF in the vicinity of the lesion, and so Fig. 9 shows a set of 438 nine depth scans taken at 1-mm intervals across the lesion. 439 The first three scans covered regions that were histologically $\mathbf{4 4 0}$ normal, and the scans were indistinguishable from those ob- 441 tained in normal tissue at remote sites. However, at the site of $\mathbf{4 4 2}$ the fourth scan, the histological structure was disrupted with 443 the cartilage zones no longer clearly identifiable, and the SHG 444 
Mansfield et al.: Collagen fiber arrangement in normal and diseased cartilage...

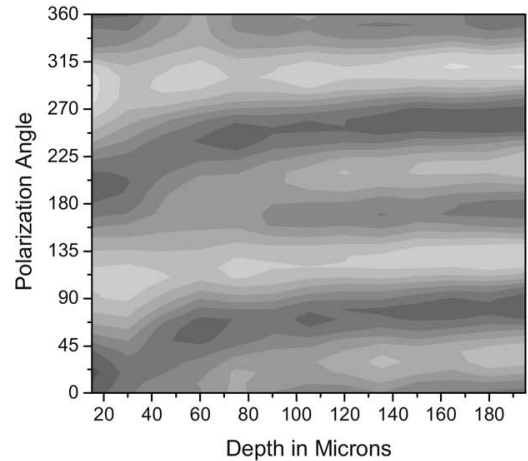

(a)

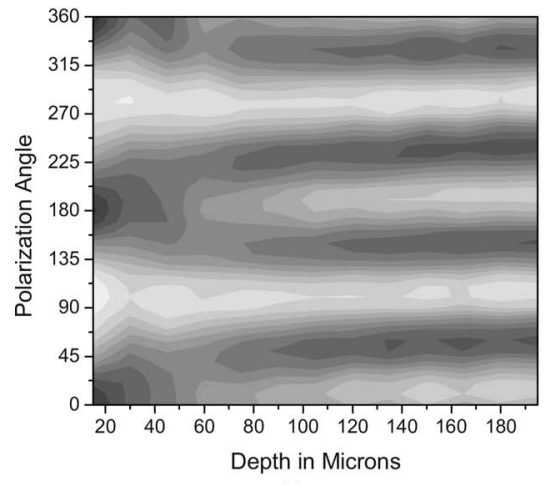

(c)

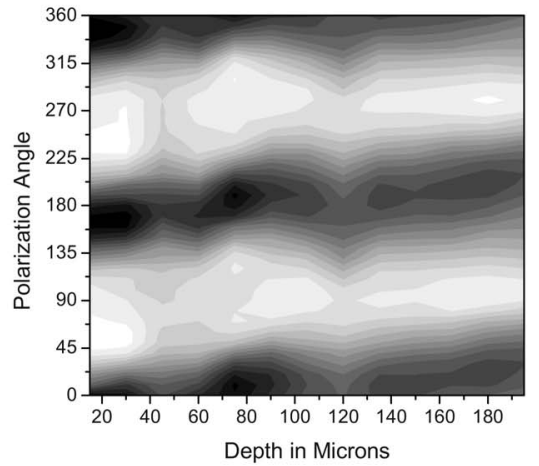

(e)

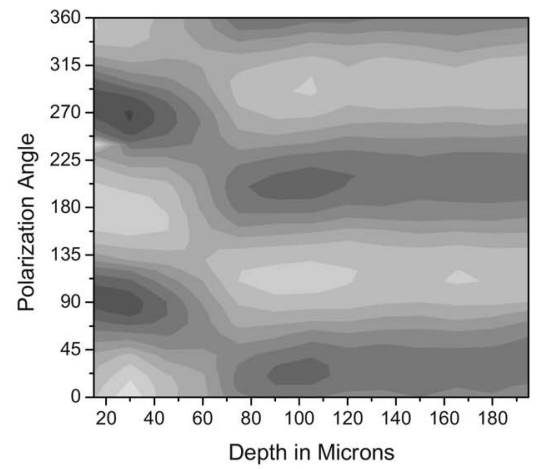

(g)
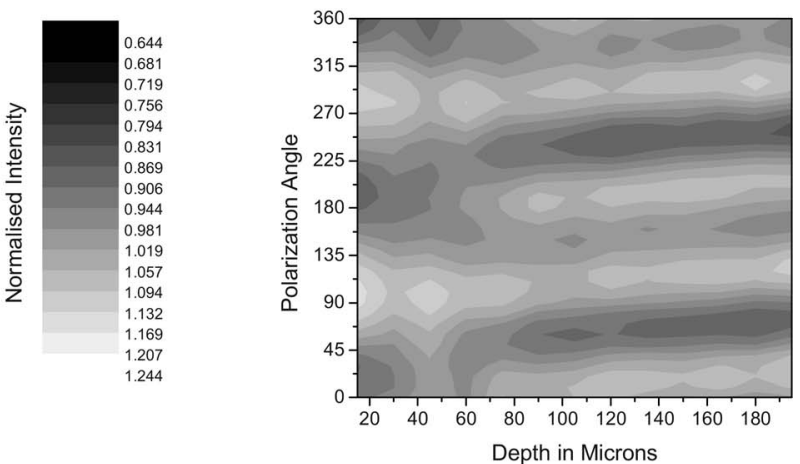

(b)

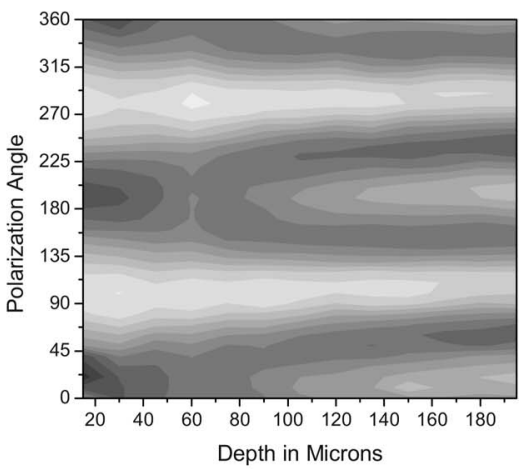

(d)

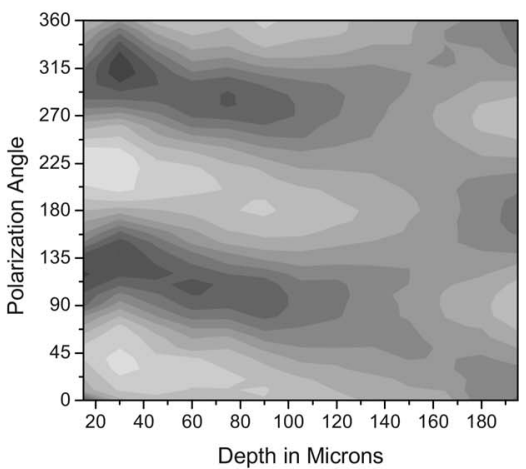

(f)

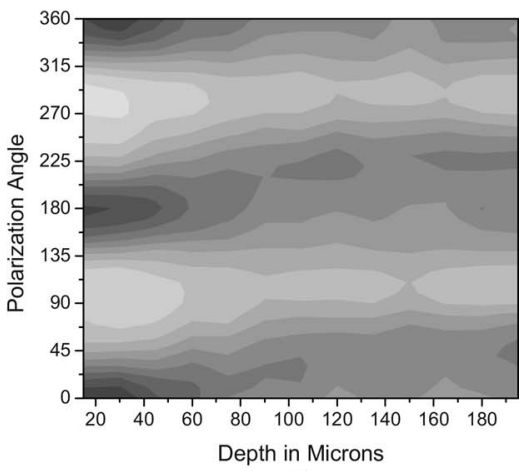

(h)

Fig. 9 Polarization dependence of SHG with depth for a series of ten sites at 1 -mm lateral positions through the lesion. Position 1 is anterior to the lesion and position 9 is posterior. At position 6, at the center of the lesion, the structure was too broken to detect any pattern of polarization sensitivity with depth. [All scales as in (a)]. 
445 pattern in the four peak pattern beginning to be lost. The 446 remaining scans were grossly abnormal in showing only two 447 intensity maxima. In addition, the angle at which the peaks in 448 intensity occurred was not constant with depth. This indicates 449 a change in the angle of the fibers, as observed in x-ray dif450 fraction studies on similar lesions. ${ }^{4}$ The intensity of the polar451 ization sensitivity was greatest in the center of the lesion. This 452 is consistent with a previous study that was able only to mea453 sure polarization sensitivity in diseased and not in healthy 454 cartilage. $^{21}$

\section{Conclusions}

456 We demonstrate that nonlinear microscopy is a useful tool 457 with which to study cartilage changes associated with disease, 458 with polarization sensitivity measurements in particular pro459 viding information on changes in collagen fiber organization. 460 The work is carried out in reflection mode, which enables 461 examination of the surface of cartilage still attached to sub462 chondral bone. Although the SHG signal from collagen is 463 propagated primarily in the forward direction, backscattering 464 was sufficiently strong to make this viable. The source of TPF 465 remains uncertain. The ratio of TPF to SHG in cartilage was 466 six times greater than that in tendon. TPF was not codistrib467 uted with the SHG, and so collagen cross links, at least some 468 of which are fluorescent, are unlikely to be the only source. 469 Elastin is a strong source of TPF, but it is present in articular 470 cartilage only in very small quantities, if at all, and certainly 471 not with the observed distribution. Proteoglycans are present 472 at much higher concentrations in cartilage than tendon, and so 473 are potential contributors to the tissue difference, but in our 474 hands, pure preparations of aggrecan monomers and aggre475 gates did not produce TPF at the wavelengths employed (data 476 not shown). Further work on this and other fundamental ques477 tions such as the mechanisms of generation of SHG in 478 collagen $^{15}$ is required if multiphoton methods are to provide 479 detailed information on tissue composition.

480 The SHG signal is polarization dependent, and surface 481 studies prove a sensitive means of detecting fiber orientation 482 in the superficial layers of cartilage. Since disruption of this 483 layer is believed to be one of the earliest indicators of disease, 484 the potential of these measurements for early in-vivo detection 485 is worth consideration. However, the interpretation of polar486 ization sensitivity data acquired from deeper regions of the 487 tissue requires care and further information. It is clear that 488 these signals are distorted by the birefringence and biattenu489 ance of the overlying tissue, and quantitative measurements of 490 these properties, together with detailed models of light propa491 gation in tissue, will be required to correct for these effects. 492 However, the detailed characterization of changes in the struc493 ture of living tissue on microscopic scales that could be ob494 tained would well reward such efforts.

495 In addition to detecting changes in the organization of the 496 intercellular matrix, multiphoton microscopy reveals changes 497 in the pericellular matrix that appear to precede changes in the 498 intercellular matrix. The ability to observe this structure in 499 living tissue should provide new avenues for studying the 500 underlying biological processes.
Acknowledgments

We would like to thank Kenton Arkill and Charlotte Moger 502 for help in collecting and preparing the tissue samples, the 503 physics department mechanical workshop for manufacturing 504 custom parts used in the microscope construction, and John 505 Hale and Alan Brady for help with the image processing. $\quad 506$

References

507

1. R. A. Stockwell, Biology of Cartilage Cells, Cambridge University 508 Press, Cambridge, UK (1979).

2. R. A. Stockwell, "Cartilage failure in osteoarthritis: relevance of nor- $\mathbf{5 1 0}$ mal structure and function. A review," Clin. Anat. 4, 161-191 (1991). 511

3. N. Ugryumova, D. P. Attenburrow, C. P. Winlove, and S. J. Matcher, 512 "The collagen structure of equine articular cartilage, characterized 513 using polarization-sensitive optical coherence tomography," J. Phys. 514 D: Appl. Phys. 38, 2612-2619 (2005).

4. C. J. Moger, R. Barrett, P. Bleuet, D. A. Bradley, R. E. Ellis, E. M. 516 Green, K. Knapp, and C. P. Winlove, "Regional variations of col- 517 lagen orientation in normal and diseased articular cartilage and sub- 518 chondral bone determined using small angle X-ray scattering 519 (SAXS)," Osteoarthritis Cartilage 15, 682-687 (2007). 520

5. N. Ugryumova, S. V. Gangnus, and S. J. Matcher, "Three- $\mathbf{5 2 1}$ dimensional optic axis determination using variable-incidence-angle $\mathbf{5 2 2}$ polarization-optical coherence tomography," Opt. Lett. 31, 2305- 523 2307 (2006).

6. W. R. Zipfel, R. M. Williams, R. Christie, A. Y. Nikitin, B. T. Hy- 525 man, and W. W. Webb, "Live tissue intrinsic emission microscopy 526 using multiphoton-excited native fluorescence and second harmonic $\mathbf{5 2 7}$ generation," Proc. Natl. Acad. Sci. U.S.A. 100, 7075-7080 (2003). 528

7. A. Zoumi, A. Yeh, and B. J. Tromberg, "Imaging cells and extracel- 529 lular matrix in vivo by using second-harmonic generation and two- 530 photon excited fluorescence," Proc. Natl. Acad. Sci. U.S.A. 99, 531 11014-11019 (2002).

8. G. Cox, E. Kable, A. Jones, I. K. Fraser, F. Manconi, and M. D. 533 Gorrell, "3-dimensional imaging of collagen using second harmonic 534 generation," J. Struct. Biol. 141, 53-62 (2003).

9. P. J. Campagnola, A. C. Millard, M. Terasaki, P. E. Hoppe, C. J. 536 Malone, and W. Mohler, "Three-dimensional high-resolution second 537 harmonic generation imaging of endogenous structural proteins in $\mathbf{5 3 8}$ biological tissues," Biophys. J. 81, 493-508 (2002).

10. J. Sun, T. Shilagard, B. Bell, M. Motamedi, and G. Vargas, "In vivo $\mathbf{5 4 0}$ multimodal nonlinear optical imaging of mucosal tissue," Opt. Ex- 541 press 12, 2478-2486 (2004).

11. R. Gauderon, P. B. Lukins, and C. J. R. Sheppard, "Optimization of $\mathbf{5 4 3}$ second-harmonic generation microscopy," Micron 32, 691-700 544 (2001).

12. L. Hsu, K. H. Kim, C. Y. Dong, P. Kaplan, T. Hancewicz, C. Buehler, 546 K. Berland, B. R. Masters, and P. T. C. So, "Two-photon imaging of 547 tissue physiology based on endogenous fluotophores," in Confocal 548 and Two-Photon Microscopy: Foundations, Applications and Ad- 549 vances, A. Diaspro, Ed., pp. 431-448, Wiley-Liss, New York (2002). 550

13. R. Richards-Kortum, R. Drezek, K. Sokolov, I. Pavlova, and M. Fol- 551 len, "Survey of Endogenous Biological Fluorophores," in Handbook 552 of Biomedical Fluorescence, M. A. Mycek and B. W. Pogue, Eds., 553 pp. 237-264, Marcel Dekker, New York (2003).

14. R. M. Williams, W. R. Zipfel, and W. W. Webb, "Interpreting second- 555 harmonic generation images of collagen I fibrils," Biophys. J. 88, 556 1377-1386 (2005).

15. P. Stoller, P. M. Celliers, K. M. Reiser, and A. M. Rubenchik, "Quan- 558 titative second-harmonic generation microscopy in collagen," Appl. 559 Opt. 42, 5209-5219 (2003).

16. I. Freund, M. Deutsch, and A. Sprecher, "Connective-tissue 561 polarity-optical 2nd-harmonic microscopy, crossed-beam summa- 562 tion, and small-angle scattering in rat-tail tendon," Biophys. J. 50, 563 693-712 (1986).

17. S. Roth and I. Freund, "Second harmonic generation in collagen," J. 565 Chem. Phys. 70, 1637-1643 (1978).

18. P. Stoller, B. M. Kim, A. M. Rubenchik, K. M. Reiser, and L. B. Da 567 Silva, "Polarization-dependent optical second-harmonic imaging of a 568 rat-tail tendon," J. Biomed. Opt. 79(2), 205-214 (2002).

19. P. Stoller, K. M. Reiser, P. M. Celliers, and A. M. Rubenchik, $\mathbf{5 7 0}$ "Polarization-modulated second harmonic generation in collagen," 571 Biophys. J. 82, 3330-3342 (2002). 
573 574

575

576

577

578

579

580

581

582

583

584

585

586

587

588

589

590

591

592
20. T. Yasui, Y. Tohno, and T. Araki, "Determination of collagen fiber orientation in human tissue by use of polarization measurement of molecular second-harmonic-generation light," Appl. Opt. 43, 28612867 (2004).

. A. T. Yeh, M. J. Hammer-Wilson, D. C. Van Sickle, H. P. Benton, A. Zoumi, B. J. Tromberg, and G. M. Peavy, "Nonlinear optical microscopy of articular cartilage," Osteoarthritis Cartilage 13, 345-352 (2005).

. T. Yasui, K. Sasaki, Y. Tohno, and T. Araki, "Tomographic imaging of collagen fiber orientation in human tissue using depth-resolved polarimetry of second-harmonic-generation light," Opt. Quantum Electron. 37, 1397-1408 (2005)

. N. J. Kemp, H. N. Zaatari, J. Park, H. G. Rylander, and T. E. Milner, "Form-biattenuance in fibrous tissues measured with polarizationsensitive optical coherence tomography (PS-OCT)," Opt. Express 13, 4611-4628 (2005).

C. W. McIlwraith, "Current Concepts in Equine Degenerative Joint Disease," J. Am. Vet. Med. Assoc. 180, 239-250 (1982).

T. A. Theodossiou, C. Thrasivoulou, C. Ekwobi, and D. L. Becker, "Second harmonic generation confocal microscopy of collagen type I from rat tendon cryosections," Biophys. J. 91, 4665-4677 (2006).

593

26. D. Eyre, "Collagen of articular cartilage," Arth. Res. 4, 30-35 (2002). 594

27. W. Horton, "Morphology of connective tissue: cartilage," in Connec- 595 tive Tissue and Its Hertiable Disorders, pp. 73-84, Wiley-Liss, New 596 York (1993)

28. V. Sankaran, J. T. Walsh, and D. J. Maitland, "Comparative study of 598 polarized light propagation in biologic tissues," J. Biomed. Opt. 7, 599 300-306 (2002)

29. J. R. Lakowicz, "Chapter 10 fluorescence anisotropy," in Principles 601 of Fluorescence Spectroscopy, L. J. R, Ed., Kluwer Scientific, New 602 York (1999).

603

30. H. Brommer, P. R. van Weeren, P. A. Brama, and A. Barneveld, 604 "Quantification and age-related distribution of articular cartilage de- 605 generation in the equine fetlock joint," Equine Vet. J. 35, 697-701 606 (2003).

607

31. J. C. Mansfield, C. P. Winlove, K. Knapp, and S. J. Matcher, "Imag- 608 ing articular cartilage using second harmonic generation 609 microscopy-art. no. 60891O," in Multiphoton Microscopy in the $610 \mathrm{AQ}$ : Biomedical Sciences VI, A. Periasamy and P. T. C. So, Eds., pp. 611 \#1 O891-0891 (2006). 
PROOF COPY 007804JBO

NOT FOR PRINT!

FOR REVIEW BY AUTHOR

NOT FOR PRINT!

AUTHOR QUERIES — 007804JBO

\#1 Author: Please supply publisher and location of publisher for Ref. 31.

PROOF COPY 007804JBO 